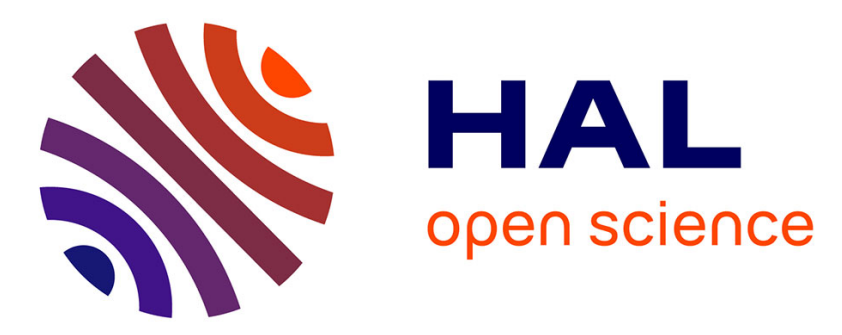

\title{
Topology design of inductors in electromagnetic casting using level-sets and second order topological derivatives
}

Alfredo Canelas, Andre Antonio Novotny, Jean Rodolphe Roche

\section{To cite this version:}

Alfredo Canelas, Andre Antonio Novotny, Jean Rodolphe Roche. Topology design of inductors in electromagnetic casting using level-sets and second order topological derivatives. Structural and Multidisciplinary Optimization, 2014, 50 (6), pp.1151-1163. 10.1007/s00158-014-1103-1 . hal-01277866

\section{HAL Id: hal-01277866 \\ https://hal.science/hal-01277866}

Submitted on 25 Feb 2016

HAL is a multi-disciplinary open access archive for the deposit and dissemination of scientific research documents, whether they are published or not. The documents may come from teaching and research institutions in France or abroad, or from public or private research centers.
L'archive ouverte pluridisciplinaire HAL, est destinée au dépôt et à la diffusion de documents scientifiques de niveau recherche, publiés ou non, émanant des établissements d'enseignement et de recherche français ou étrangers, des laboratoires publics ou privés. 


\title{
Topology design of inductors in electromagnetic casting using level-sets and second order topological derivatives
}

\author{
Alfredo Canelas · Antonio A. Novotny · Jean R. Roche
}

\begin{abstract}
We propose a new iterative method for the topology design of the inductors in electromagnetic casting. The method is based on a level-set representation of the solution together with first and second order topological derivatives. The optimal design is found by minimizing a Kohn-Vogelius-type functional for the problem. The complete topological expansion of the objective functional, which is herein given, is used to define the iterative step. Results for several numerical examples show that the technique proposed can be efficiently used in the design of suitable inductors.
\end{abstract}

Keywords Topological asymptotic analysis - Topological derivatives - Inverse problem - Electromagnetic casting

\section{Introduction}

The design problem in electromagnetic casting is the determination of the electrical currents that shape certain mass of liquid metal into a predefined geometry, the target

Preliminary ideas and results presented in Canelas et al. (2012)

A. Canelas (区)

Instituto de Estructuras y Transporte, Facultad de Ingeniería, Universidad de la República, Julio Herrera y Reissig 565, Montevideo, Uruguay

e-mail: acanelas@ fing.edu.uy

\author{
A. A. Novotny \\ Laboratório Nacional de Computação Científica LNCC/MCT, \\ Av. Getúlio Vargas 333, 25651-075, Petrópolis, RJ, Brazil \\ J. R. Roche \\ Institut Elie Cartan de Lorraine, Université de Lorraine, CNRS, \\ INRIA, B.P. 70239, 54506, Vandoeuvre lès Nancy, France
}

shape. In previous papers we studied this problem considering inductors made of single small solid-core wires (Canelas et al. 2009a), and large inductors made of bundled insulated strands (Canelas et al. 2009b). In both cases the number of inductors was fixed in advance. In a recent paper we overcome this constraint, and looked for configurations of inductors considering different topologies with the purpose of obtaining inductors with more realistic geometric configurations (Canelas et al. 2011). A different topology optimization approach for locating small solid-core wires has recently been proposed by Shin et al. (2012).

In the present paper we formulate the design problem as an optimization problem by means of a shape functional based on the Kohn-Vogelius criterion (Roche 1996; Friedman and Vogelius 1989; Kohn and Vogelius 1984; Brühl et al. 2003; Eppler and Harbrecht 2010). The main achievements of the present paper are the following: First, we give the analytical expression of the complete topological asymptotic expansion of the Kohn-Vogelius shape functional, generalizing the results of Canelas et al. (2011) and proving that the expansion has actually a finite number of terms. Second, we use this topological asymptotic expansion to devise an efficient topology optimization algorithm based on the level-set technique proposed by Amstutz and Andrä (2006). The novelty of this paper is that we propose a topology optimization algorithm using non-standard level set method together with first and second order topological derivatives.

The contents of this paper are organized as following. Section 2 describes the direct free-surface problem concerning the electromagnetic casting and formulates the problem of designing suitable inductors as a problem of minimization of a Kohn-Vogelius-type objective functional. Section 3 introduces the topological derivative concept and states the asymptotic expansion of the Kohn-Vogelius 
functional. The numerical method is presented in Section 4. Some examples are presented in Section 5, to show that the method proposed can efficiently find suitable designs. The conclusions of this paper are presented in Section 6 .

\section{The electromagnetic casting problem}

The electromagnetic casting is an industrial technique used in preparation of very pure samples, preparation of aluminum ingots using soft-contact confinement of the liquid metal, shaping of components of aeronautical engines made of superalloy materials $(\mathrm{Ni}, \mathrm{Ti}, \ldots$ ), etc. (Zhiqiang et al. 2002; Fu et al. 2004).

In this paper we study a vertical column of liquid metal falling down into an electromagnetic field created by vertical inductors. We assume that a stationary horizontal section is reached, so that the two-dimensional model is valid, and that the frequency of the imposed current is very high, so that the magnetic field does not penetrate into the metal (skin effect); see (Jackson 1998; Brancher and SéroGuillaume 1985; Gagnoud et al. 1986; Henrot and Pierre 1989; Moffatt 1985; Novruzi and Roche 1995; Pierre and Roche 1991; Shercliff 1981).

The exterior boundary value problem regarding the magnetic field in terms of the flux function $\varphi: \Omega \rightarrow \mathbb{R}$ is:

$$
\left\{\begin{aligned}
-\Delta \varphi & =\mu_{0} j_{0} & & \text { in } \Omega, \\
\varphi & =0 & & \text { on } \Gamma, \\
\varphi(x) & =c+o(1) & & \text { as }\|x\| \rightarrow \infty
\end{aligned}\right.
$$

where $\Omega \subset \mathbb{R}^{2}$ is the exterior of the compact, simply connected and with a non-void interior, domain $\omega$ occupied by the cross-section of the metal column. In (1) $\Gamma$ is the boundary of $\Omega$, that we assume is at least of class $C^{2}$. By $\|\cdot\|$ we denote the Euclidean norm, the little-o notation means $\lim _{\|x\| \rightarrow \infty} o(f(x)) / f(x)=0, c \in \mathbb{R}$ is the value at infinity of the solution $\varphi$, which is also an unknown of (1) (Canelas et al. 2009a, 2009b, 2011), $\mu_{0}$ is the vacuum permeability, and $j_{0}$ is the vertical component of the electric current density vector, which is assumed compactly supported in $\boldsymbol{\Omega}$ and such that the total current is zero:

$\int_{\Omega} j_{0} d x=0$

Problem (1) has unique solutions $\varphi \in W_{0}^{1}(\Omega)$ and $c \in \mathbb{R}$ (Nédélec 2001; Atkinson 1997), where $W_{0}^{1}(\Omega)$ is defined as:

$$
W_{0}^{1}(\boldsymbol{\Omega})=\left\{u: \rho u \in L^{2}(\boldsymbol{\Omega}) \text { and } \nabla u \in L^{2}(\boldsymbol{\Omega})\right\},
$$

with $\rho(x)=\left[\sqrt{1+\|x\|^{2}} \log \left(2+\|x\|^{2}\right)\right]^{-1}$. The equilibrium of the liquid metal boundary is given by (Pierre and Roche 1991, 1993, 1997, 2005; Novruzi and Roche 2000):

$\frac{1}{2 \mu_{0}}\left|\frac{\partial \varphi}{\partial n}\right|^{2}+\sigma \mathcal{C}=p_{0} \quad$ on $\Gamma$,

where $n$ is the outward-pointing unit normal vector of $\Gamma$, $\mathcal{C}$ is the curvature of $\Gamma$ seen from the metal, $\sigma$ is the surface tension of the liquid and the constant $p_{0}$ is an unknown of the problem. Physically, $p_{0}$ represents the difference between the internal and external pressures.

In the free-surface problem of electromagnetic casting the electric current density $j_{0}$ is given, and one needs to find the shape $\omega$, having a given area $S_{0}=\int_{\omega} d x$, such that the flux $\varphi$, solution to (1), also satisfies the equilibrium (4) for some real constant $p_{0}$.

\subsection{The design problem}

In the design problem we have to determine the current density $j_{0}$ satisfying (2) such that the solution $\varphi$ of (1) also satisfies the equilibrium (4). It is known that at the equilibrium $p_{0}=\max _{\Gamma} \sigma \mathcal{C}$ (Henrot and Pierre 1989; Felici and Brancher 1991; Canelas et al. 2011). Therefore, for a given target shape with bounded curvature, $p_{0}$ can be calculated. Defining $\bar{p}=\sqrt{2 \mu_{0}\left(p_{0}-\sigma \mathcal{C}\right)}$, the equilibrium equation in terms of the flux function reads

$\frac{\partial \varphi}{\partial n}=\varkappa \bar{p} \quad$ on $\Gamma$,

where $\varkappa= \pm 1$, has the changes of sign located at points where the curvature of $\Gamma$ is a global maximum. The two possible ways of defining $\varkappa$ lead to the same solution $j_{0}$ but with the opposite sign (Canelas et al. 2011). From (5) we have that a necessary condition for the existence of a solution is the following (Canelas et al. 2011):

$\int_{\Gamma} \varkappa \bar{p} d s=0$

It is known that the design problem is inherently ill posed: small variation of the liquid boundary may cause dramatic variations in the solution jo (Henrot and Pierre 1989; Felici and Brancher 1991). In addition, the uniqueness of the solution in terms of $j_{0}$ cannot be ensured, see a formal proof in (Shin et al. 2012) and also (Henrot and Pierre 1989; Canelas et al. 2011). Therefore, we formulate the design problem as an optimization problem, looking for a solution (maybe just an approximate solution) that minimizes the following shape functional based on the Kohn-Vogelius criterion:

$\psi(0)=J(\phi)=\frac{1}{2}\|\phi\|_{L^{2}(\Gamma)}^{2}=\frac{1}{2} \int_{\Gamma} \phi^{2} d s$, 
where the auxiliary function $\phi \in W_{0}^{1}(\Omega)$ depends implicitly on $j_{0}$ and $c$ by solving the following boundary-value problem

$$
\left\{\begin{aligned}
-\Delta \phi & =\mu_{0} j_{0} & & \text { in } \boldsymbol{\Omega}, \\
\frac{\partial \phi}{\partial n} & =\varkappa \bar{p}-d\left(j_{0}\right) & & \text { on } \Gamma, \\
\phi(x) & =c+o(1) & & \text { as }\|x\| \rightarrow \infty,
\end{aligned}\right.
$$

where, denoting $|\Gamma|=\int_{\Gamma} d s, d\left(j_{0}\right)$ is defined as

$$
d\left(j_{0}\right)=|\Gamma|^{-1} \int_{\Omega} \mu_{0} j_{0} d x
$$

The term $d\left(j_{0}\right)$ is introduced in (8) to correctly define $\phi$ for $j_{0}$ not necessarily satisfying condition (2). In fact, given some fixed value $c$, and assuming that (6) is satisfied, we note that problem (8) has a unique solution in $W_{0}^{1}(\Omega)$, see (Atkinson 1997; Hsiao and Wendland 2008).

In this paper we assume that condition (6) is satisfied, and look for $j_{0}$ satisfying (2) and a constant $c$ such that the solution $\phi$ to (8) minimizes the shape functional (7). Note that the minimum is attained if $\phi \equiv 0$ on $\Gamma$. In this case, from the well-posedness of problems (1) and (8), we have $\phi \equiv \varphi$ in $\Omega$. We can easily eliminate the variable $c$ of the optimization problem by defining it as the global minimum $c^{*}\left(j_{0}\right)$ of $(7)$ for each fixed $j_{0}$, i.e., we take $c=c^{*}\left(j_{0}\right)=\arg \min _{c} J\left(\phi\left(j_{0}, c\right)\right)$ (Canelas et al. 2011). Hence we can formulate an equivalent optimization problem as follows: minimize the shape functional (7), where $\phi \in W_{0}^{1}(\boldsymbol{\Omega})$ depends implicitly on $j_{0}$ only, by solving the following problem:

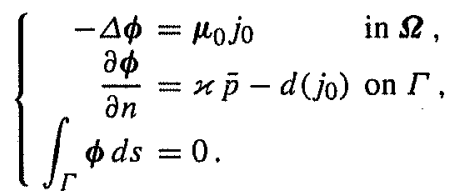

Problem (10) is well-posed, as rigorously stated in the following lemma.

Lemma 1 Given $b \in L_{2}(\boldsymbol{\Omega}), q \in L_{2}(\Gamma)$ and $c \in \mathbb{R}$, satisfying the compatibility condition $\int_{\Omega} b d x+\int_{\Gamma} q d s=0$, there is a unique solution $\phi \in W_{0}^{1}(\Omega)$ to the problem

$$
\left\{\begin{aligned}
-\Delta \phi & =b \text { in } \Omega \\
\frac{\partial \phi}{\partial n} & =q \text { on } \Gamma \\
\int_{\Gamma} \phi d s & =c
\end{aligned}\right.
$$

which depends continuously on the problem data, i.e., there is $C \in \mathbb{R}$ such that $\|\phi\|_{W_{0}^{1}(\Omega)} \leq C\left(b_{L_{2}(\Omega)}+\|q\|_{L_{2}(\Gamma)}+|c|\right)$.
Proof Let $\xi$ be the solution in $W_{0}^{1}(\Omega)$ of:

$\left\{\begin{aligned}-\Delta \xi & =b & & \text { in } \Omega, \\ \frac{\partial \xi}{\partial n} & =q & & \text { on } \Gamma, \\ \xi(x) & =o(1) & & \text { as }\|x\| \rightarrow \infty .\end{aligned}\right.$

In the space $\left\{u \in W_{0}^{1}(\Omega): u=o(1)\right.$ as $\left.\|x\| \rightarrow \infty\right\}$, the map $u \mapsto\|\nabla u\|_{L_{2}(\Omega)}$ is a norm equivalent to $\|\cdot\|_{W_{0}^{1}(\Omega)}$ (Nédélec 2001; Giroire 1976). Therefore, the standard analysis of the variational formulation of problem (12) shows that there is a unique solution $\xi$ satisfying $\|\xi\|_{W_{0}^{1}(\Omega)} \leq$ $C_{1}\left(\|b\|_{L_{2}(\Omega)}+\|q\|_{L_{2}(\Gamma)}\right)$ for some real $C_{1}$, see (Hsiao and Wendland 2008; Atkinson 1997; Nédélec 2001; Giroire 1976). The proof of the lemma is promptly obtained using this result, considering that $\phi=\xi+|\Gamma|^{-1}\left(c-\int_{\Gamma} \xi d s\right)$.

\section{Topological derivative concept}

The topological derivative measures the sensitivity of a given shape functional with respect to an infinitesimal singular domain perturbation, such as insertion of holes, inclusions, source-terms or even cracks (Eschenauer et al, 1994; Sokołowski and Żochowski 1999; Céa et al. 2000). It has proved extremely useful in the treatment of a wide range of problems, see (Amstutz and Andrä 2006; Amstutz et al. 2010; Amstutz and Novotny 2010; Novotny et al. 2007; Amstutz et al. 2005; Feijóo 2004; Guzina and Bonnet 2006; Hintermüller and Laurain 2008; Hintermüller et al. 2012; Belaid et al. 2008; Hintermüller and Laurain 2009; Larrabide et al. 2008). Concerning the theoretical development of the topological asymptotic analysis, see the book by Novotny and Sokołowski J (2013) and the papers (Amstutz (2006); de Faria and Novotny 2009; Garreau et al. 2001; Nazarov 2003; Sokołowski and Żochowski 2003). See also the books by Ammari and Kang (2004) and Ammari and Kang (2007) regarding the asymptotic analysis of PDE solutions and their applications to inverse problems.

Consider that a domain $\Omega$ is subject to a non-smooth perturbation confined in a small ball $B_{\varepsilon}(\hat{x})$ of radius $\varepsilon$ and center $\widehat{x} \in \boldsymbol{\Omega}$. Then, if a given shape functional $\psi(\varepsilon)$, associated to the topologically perturbed domain, admits the expansion (Sokołowski and Żochowski 1999)

$\psi(\varepsilon)=\psi(0)+f_{1}(\varepsilon) D_{T}^{1} \psi+f_{2}(\varepsilon) D_{T}^{2} \psi+o\left(f_{2}(\varepsilon)\right)$,

where $\psi(0)$ is the shape functional value for the unperturbed domain and $f_{i}(\varepsilon), 1 \leq i \leq 2$, are positive functions such that $f_{i}(\varepsilon) \rightarrow 0$, and $f_{2}(\varepsilon) / f_{1}(\varepsilon) \rightarrow 0$, when $\varepsilon \rightarrow 0$, we say that the functions $\widehat{x} \mapsto D_{T}^{i} \psi(\widehat{x}), 1 \leq i \leq 2$, are the topological derivatives of $\psi$ at $\widehat{x}$. The term $f_{1}(\varepsilon) D_{T}^{1} \psi+$ $f_{2}(\varepsilon) D_{T}^{2} \psi$ can be seen as a second order correction of $\psi(0)$ to approximate $\psi(\varepsilon)$. 


\subsection{The topological derivatives calculation}

Associated to the solution $\phi$ of (10), we define the function $\phi_{\varepsilon} \in W_{0}^{1}(\Omega)$ being the solution to the following problem:

$\left\{\begin{array}{rlrl}-\Delta \phi_{\varepsilon} & =\mu_{0} j_{\varepsilon} & & \text { in } \boldsymbol{\Omega}, \\ \frac{\partial \phi_{\varepsilon}}{\partial n} & =\varkappa \bar{p}-d\left(j_{\varepsilon}\right) \text { on } \Gamma, \\ \int_{\Gamma} \phi_{\varepsilon} d s & =0 .\end{array}\right.$

where the perturbed electric current density $j_{\varepsilon}$ is identical to $j_{0}$ everywhere in $\boldsymbol{\Omega}$ except in $B_{\varepsilon}(\widehat{x}) \subset \boldsymbol{\Omega}$, a small ball of radius $\varepsilon$ and center $\widehat{x}$. More precisely, $j_{\varepsilon}=j_{0}+\alpha I \chi_{B_{\varepsilon}(\widehat{x})}$, where $I$ is a given current density value and $\alpha= \pm 1$ is the sign of the current density in $B_{\varepsilon}(\widehat{x})$.

The shape functional associated to the perturbed problem reads:

$\psi(\varepsilon)=J\left(\phi_{\varepsilon}\right)=\frac{1}{2} \int_{\Gamma} \phi_{\varepsilon}^{2} d s$

Let $u^{*}$ be the fundamental solution of the Laplace operator:

$u^{*}(y, x)=-\frac{1}{2 \pi} \ln \|y-x\|$.

Theorem 1 For $\widehat{x} \in \Omega$, there exists $\varepsilon_{0}$ such that for all $\varepsilon<\varepsilon_{0}$ the following equality holds

$\psi(\varepsilon)=\psi(0)+f_{1}(\varepsilon) D_{T}^{1} \psi+f_{2}(\varepsilon) D_{T}^{2} \psi$,

with the functions $f_{1}(\varepsilon)=\pi \varepsilon^{2}, f_{2}(\varepsilon)=\pi^{2} \varepsilon^{4}$ and the topological derivatives

$D_{T}^{1} \psi(\widehat{x})=\alpha \mu_{0} I \int_{\Gamma} \phi \vec{f}_{\widehat{x}} d s$,

$D_{T}^{2} \psi(\widehat{x})=\frac{1}{2} \mu_{0}^{2} I^{2} \int_{\Gamma} \vec{f}_{\widehat{x}}^{2} d s$.

In (18)-(19), $\vec{f}_{\widehat{x}}$ is a continuous function on $\Gamma$, given by

$\bar{f}_{\widehat{x}}(x)=u^{*}(\widehat{x}, x)-u^{*}(\bar{x}, x)+g_{\widehat{x}}(x) \quad \forall x \in \Gamma$,

where $\vec{x}$ is a fixed interior point of $\omega$ and $g_{\hat{x}} \in W_{0}^{1}(\Omega)$ is solution to:

$\left\{\begin{aligned}-\Delta g_{\widehat{x}} & =0 \\ \frac{\partial g_{\widehat{x}}}{\partial n} & =\frac{\partial u^{*}(\bar{x}, \cdot)}{\partial n}-\frac{\partial u^{*}(\widehat{x}, \cdot)}{\partial n}-|\Gamma|^{-1} \text { on } \Gamma, \\ \int_{\Gamma} g_{\widehat{x}} d s & =\int_{\Gamma} u^{*}(\bar{x}, \cdot)-u^{*}(\widehat{x}, \cdot) d s .\end{aligned}\right.$

Moreover, the topological derivatives $D_{T}^{1} \psi$ and $D_{T}^{2} \psi$ are Lipschitz continuous functions with respect to their argur ment $\widehat{x}$ in any compact $\mathcal{D} \subset \boldsymbol{\Omega}$.

Proof Let $\varepsilon_{0}$ be small enough such that every point $x \in$ $\Gamma$ is outside the ball $B_{\varepsilon_{0}}(\widehat{x})$. For some $\varepsilon<\varepsilon_{0}$, set $f_{\widehat{x}}=$ $\left(\alpha \mu_{0} I \pi \varepsilon^{2}\right)^{-1}\left(\phi_{\varepsilon}-\phi\right) \in W_{0}^{1}(\Omega)$. Taking into account (10),
(14), (22) and the fact that $d\left(j_{\varepsilon}\right)=d\left(j_{0}\right)+|\Gamma|^{-1} \alpha \mu_{0} I \pi \varepsilon^{2}$, we have that $f_{\widehat{x}}$ solves:

$\left\{\begin{array}{rlrl}-\Delta f_{\widehat{x}} & =\left(\pi \varepsilon^{2}\right)^{-1} \chi_{B_{\varepsilon}(\widehat{x})} & \text { in } \Omega, \\ \frac{\partial f_{\widehat{x}}}{\partial n} & =-|\Gamma|^{-1} & \text { on } \Gamma, \\ \int_{\Gamma} f_{\widehat{x}} d s & =0 .\end{array}\right.$

A particular solution to (22) is $f_{\bar{x}}^{p}$ given by

$f_{\bar{x}}^{p}(x)=\left(\pi \varepsilon^{2}\right)^{-1} \int_{B_{\varepsilon}(\tilde{x})} u^{*}(y, x) d y-u^{*}(\bar{x}, x)$

This last expression can be integrated exactly for each point $x$ outside the ball $B_{\varepsilon}(\widehat{x})$. In particular, for each $x \in \Gamma$ we have $f_{\widehat{x}}^{p}(x)=u^{*}(\widehat{x}, x)-u^{*}(\bar{x}, x)$. Then, the solution of (22) is $f_{\widehat{x}}=f_{\widehat{x}}^{p}+g_{\hat{x}}$ where $g_{\hat{x}}$ is the solution to (21). We now take $\hat{f}_{\widehat{x}}=\left.f_{\widehat{x}}\right|_{\Gamma}$ and (20) holds. Since $g \widehat{x}$ does not depend on $\varepsilon,(20)$ shows that $\bar{f}_{\widehat{x}}$ does not depend on $\varepsilon$ too. Since $\Gamma$ is of class $C^{2}$, and the boundary data in (21) is continuous, $g_{\widehat{x}} \in C^{1}(\bar{\Omega})$ (Atkinson 1997). Hence, $g_{\widehat{x}} \mid \Gamma$ and then $\bar{f}_{\widehat{x}}$ are continuous. The complete topological asymptotic expansion of the shape functional becomes

$$
\begin{aligned}
\psi(\varepsilon) & =J\left(\phi_{\varepsilon}\right)=\frac{1}{2} \int_{\Gamma}\left(\phi+\alpha \mu_{0} I \pi \varepsilon^{2} f_{\widehat{x}}\right)^{2} d s \\
& =\psi(0)+\pi \varepsilon^{2}\left(\alpha \mu_{0} I \int_{\Gamma} \phi \bar{f}_{\widehat{x}} d s\right)+ \\
& +\pi^{2} \varepsilon^{4}\left(\frac{1}{2} \mu_{0}^{2} I^{2} \int_{\Gamma} \bar{f}_{\widehat{x}}^{2} d s\right),
\end{aligned}
$$

where the expressions inside the parentheses depend on $\widehat{x}$ but do not depend on $\varepsilon$, hence being the first and second order topological derivatives of $\psi$. To show that these derivatives are Lipschitz in $\mathcal{D}$, the Cauchy-Schwartz inequality applied to (18)-(19) gives

$$
\left|D_{T}^{1} \psi(\widehat{x})-D_{T}^{1} \psi(\widehat{y})\right| \leq \mu_{0} I\|\phi\|_{L_{2}(\Gamma)}\left\|f_{\widehat{x}}-f_{\widehat{y}}\right\|_{L_{2}(\Gamma)},
$$

$$
\begin{aligned}
\left|D_{T}^{2} \psi(\widehat{x})-D_{T}^{2} \psi(\widehat{y})\right| \leq & \frac{1}{2} \mu_{0}^{2} I^{2}\left\|f_{\widehat{x}}+f_{\widehat{y}}\right\|_{L_{2}(\Gamma)} \| f_{\widehat{x}} \\
& -f_{\widehat{y}} \|_{L_{2}(\Gamma)} .
\end{aligned}
$$

Note that the existence of the norms in (25)-(26) is ensured by the trace theorem applied to the functions $\phi, f_{\widehat{x}}$ and $f_{\widehat{y}}$ of $W_{0}^{1}(\Omega)$. Then, since $\left\|f_{\widehat{x}}+f_{\widehat{y}}\right\|_{L_{2}(\Gamma)} \leq\left\|f_{\widehat{x}}\right\|_{L_{2}(\Gamma)}+$ $\left\|f_{\widehat{y}}\right\|_{L_{2}(\Gamma)}$, the continuity property follows from

(i) $\left\|f_{\widehat{x}}\right\|_{L_{2}(\Gamma)}$ is bounded in $\mathcal{D}$,

(ii) $\left\|f_{\widehat{x}}-f_{\widehat{y}}\right\|_{L_{2}(I)} \leq C\|\widehat{x}-\widehat{y}\|$ for some $C \in \mathbb{R}$, with $\widehat{x}, \widehat{y} \in \mathcal{D}$. 
Note that (ii) implies the continuity of the map $\widehat{x} \mapsto f_{\widehat{x}} \mid \Gamma$, for the norm $\|\cdot\|_{L_{2}(\Gamma)}$, so that (ii) implies (i). To prove (ii) we use (20) to obtain

$$
\begin{gathered}
\left\|\bar{f}_{\widehat{x}}-\bar{f}_{\hat{y}}\right\|_{L_{2}(\Gamma)} \leq\|u(\widehat{x}, \cdot)-u(\widehat{y}, \cdot)\|_{L_{2}(\Gamma)} \\
+\left\|g_{\widehat{x}}-g \widehat{y}\right\|_{L_{2}(\Gamma)},
\end{gathered}
$$

where $g_{\widehat{x}}-g_{\widehat{y}} \in W_{0}^{1}(\Omega)$ is solution to

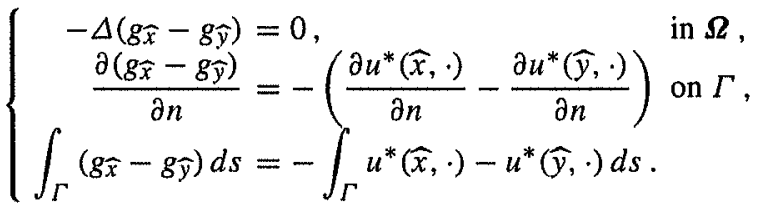

By the trace theorem we have $\left\|g_{\widehat{x}}-g_{\hat{y}}\right\|_{L_{2}(\Gamma)} \leq C_{1} \| g_{\widehat{x}}-$ $g \hat{y} \|_{W_{0}^{1}(\Omega)}$ for some $C_{1} \in \mathbb{R}$. Hence (27) and Lemma 1 applied to (28) give

$$
\begin{aligned}
\left\|\bar{f}_{\widehat{x}}-\bar{f}_{\hat{y}}\right\|_{L_{2}(\Gamma)} & \leq C_{2}\|u(\widehat{x}, \cdot)-u(\widehat{y}, \cdot)\|_{L_{2}(\Gamma)}+ \\
& +C_{3}\left\|\frac{\partial u^{*}(\widehat{x}, \cdot)}{\partial n}-\frac{\partial u^{*}(\widehat{y}, \cdot)}{\partial n}\right\|_{L_{2}(\Gamma)},
\end{aligned}
$$

for some $C_{2}, C_{3} \in \mathbb{R}$. Result (ii) is obtained from the previous inequality and the fact that $\Gamma \in C^{2}$, so that $u^{*}$ and $\partial u^{*} / \partial n$ are continuous and differentiable with respect to both variables in the compact $\mathcal{D} \times \Gamma$, hence they are Lipschitz in $\mathcal{D} \times \Gamma$.

Instead of using (18), the first order topological derivative can be computed efficiently by using the adjoint state $v$, see (Canelas et al. 2011),

$D_{T}^{1} \psi(\widehat{x})=-\alpha \mu_{0} I v(\widehat{x})$,

where $v$ is the unique solution in $W_{0}^{1}(\Omega)$ to the following problem:

$\left\{\begin{aligned}-\Delta v & =0 \quad \text { in } \Omega \\ \frac{\partial v}{\partial n} & =-\phi \text { on } \Gamma, \\ \int_{\Gamma} v d s & =0 .\end{aligned}\right.$

Remark 1 Note that (30) allows us to compute the first order topological derivative at several points by solving once the boundary value problem (31). For the second order derivatives, the boundary value problem (21) must be solved once for each point $\widehat{x}$. However, note that unlike the solution to (31), the solution to (21) does not depend on $\phi$, and then it does not depend on the actual current density distribution $j_{0}$. Therefore, second order derivatives can be computed once before starting the optimization process. In addition, the point $\widehat{x}$ only affects the right hand side of the linear systems of the numerical approach, thus evaluation of second order derivatives requires of only one factorization. Hence, the computational cost of evaluation of second order derivatives is much lower than the usual case where they must be computed at each iteration, requiring factorization of different coefficient matrices.

We end this section with the following result:

Theorem 2 Givena centrally symmetric domain $\omega$, i.e. simmetric with respect to the center $\bar{x}$ interior to $\omega$, then the topological derivative $D_{T}^{2} \psi(x)>0$ for all $x \in \Omega$.

Proof According to (19), $D_{T}^{2} \psi(x) \geq 0$, for all $x \in \Omega$ and hence we just have to prove that there is no point $\widehat{x} \in \Omega$ such that $D_{T}^{2} \psi(\widehat{x})=0$. Let us assume that a point $\widehat{x} \in \Omega$ satisfying $D_{T}^{2} \psi(\widehat{x})=0$ exists. Hence, according to (19), the solution $f$ of (22) satisfies $f(x)=0$ for all $x \in \Gamma$. We know that there exists a constant $c$ such that the solution $g_{\widehat{x}}$ to (21) satisfies the growth condition $g_{\widehat{x}}(x)=c+o(1)$ (Atkinson 1997). Therefore, since outside $B_{\varepsilon}(\hat{x})$ we have $f_{0}(x)=u^{*}(\widehat{x}, x)-u^{*}(\bar{x}, x), f$ satisfies the same growth condition for the same $c$. Therefore, $f$ should be a solution in $W_{0}^{1}(\Omega)$ to the following problem:

$$
\left\{\begin{aligned}
-\Delta f & =\left(\pi \varepsilon^{2}\right)^{-1} \chi B_{\varepsilon}(\widehat{x}) & & \text { in } \Omega, \\
f & =0 & & \text { on } \Gamma, \\
\frac{\partial f}{\partial n} & =-|\Gamma|^{-1} & & \text { on } \Gamma, \\
f(x) & =c+o(1) & & \text { as }\|x\| \rightarrow \infty .
\end{aligned}\right.
$$

On the other hand, due to the growth condition satisfied by $f$, the following boundary integral equation holds for each function $q: \Omega \rightarrow \mathbb{R}$ satisfying $\Delta q(x)=0$ in $\Omega$ and $q(x)=$ $o(1)$ as $\|x\| \rightarrow \infty$ :

$\int_{B_{\varepsilon}(\hat{x})}\left(\pi \varepsilon^{2}\right)^{-1} q(x) d x=\int_{\Gamma} f \frac{\partial q}{\partial n} d s-\int_{\Gamma} \frac{\partial f}{\partial n} q d s$.

Since $\widehat{x}$ is the center of $B_{\varepsilon}(\widehat{x}) \subset \Omega$, by using the mean value property of harmonic functions, and by replacing the boundary values of $f$ into (33) we obtain

$q(\widehat{x})=|\Gamma|^{-1} \int_{\Gamma} q d s$.

Consider a Cartesian coordinate system with $\bar{x}$ as its origin. Let $\left(x_{1}, x_{2}\right)$ be the coordinates of $x \in \Omega$ and let the functions $q_{1}$ and $q_{2}$ be defined as

$q_{1}(x)=\frac{x_{1}}{r^{2}}, \quad q_{2}(x)=\frac{x_{2}}{r^{2}}$,

with $r^{2}=x_{1}^{2}+x_{2}^{2}$. Since $q_{1}$ and $q_{2}$ satisfy the hypothesis required for $q$, (44) gives for these functions

$\frac{\widehat{x_{1}}}{\widehat{r}^{2}}=|\Gamma|^{-1} \int_{\Gamma} \frac{x_{1}}{r^{2}} d s, \quad \frac{\widehat{x}_{2}}{\widehat{r}^{2}}=|\Gamma|^{-1} \int_{\Gamma} \frac{x_{1}}{r^{2}} d s$,

where $\left(\widehat{x}_{1}, \widehat{x}_{2}\right)$ are the coordinates of $\widehat{x}$ and $\widehat{r}^{2}=\widehat{x}_{1}^{2}+\widehat{x}_{2}^{2}$. By hypothesis $\widehat{x} \in \Omega$, hence $\widehat{r}^{2}>0$ and at least one of $q_{1}(\hat{x})$ and $q_{2}(\widehat{x})$ is non-zero. However, both integral expressions of (36) vanish because of the central symmetry of $\Gamma$. Since 
there is a contradiction, a point $\widehat{x} \in \Omega$ satisfying $D_{T}^{2} \psi(\widehat{x})=$ 0 does not exist.

\section{A topological derivative-based level-set algorithm}

In this section we state a level-set topology design algorithm based on the topological derivatives obtained in the previous section. First we consider domains $\boldsymbol{\Omega}$ which possess central symmetry. In this case we can devise a level set algorithm to generate a sequence of current density functions $j_{0}$ satisfying (2) at each iteration. In the general case, condition (2) can not be ensured automatically, and a penalty function strategy to enforce satisfaction of this constraint will be followed.

\subsection{The symmetric case}

In this case the current density $j_{0}$ is sought as the solution of the general optimization problem stated as

Minimize $J(\phi)$$$
x_{0} \in \mathrm{O}
$$

$\int_{\Omega} j_{0} d x=0$

where $\mathcal{O}$ is the set of functions $j_{0}=I\left(\chi_{\Theta^{+}}-\chi_{\Theta^{-}}\right)$with $\chi_{\Theta^{+}}$and $\chi_{\Theta^{-}}$being the characteristic functions of the sets $\boldsymbol{\Theta}^{+}$and $\boldsymbol{\Theta}^{-}$, which are the open and disjoints parts of $\boldsymbol{\Omega}$ representing the regions where the current density $j_{0}$ is, respectively, positive or negative. We assume that $\Theta^{+}$and $\Theta^{-}$are in a compact $\Theta \subset \Omega$. The function $\phi$ is the solution of $(8)$ in $W_{0}^{1}(\boldsymbol{\Omega})$. Therefore, the design variables of problem (37) are the shape and topology of $\Theta^{+}$and $\Theta^{-}$.

According to the topological expansion (24), introduction of a circular region of current density $\alpha I, \alpha \in$ $\{+1,-1\}$, and center $\widehat{x} \in \boldsymbol{\Omega}$ changes the value of the objective function of problem (37). If we consider an optimal configuration of inductors with respect to the class of perturbations we are analyzing, the introduction of that small circular region at $\hat{x} \in \Omega^{0}=\Omega \backslash\left(\overline{\Theta^{+}} \cup \overline{\Theta^{-}}\right)$should not increase the objective function. Hence, according to the expression (30) of the first order topological derivative, the optimal configuration should satisfy the following necessary condition:

$-\alpha \mu_{0} I v(\widehat{x}) \geq 0 \quad \forall \widehat{x} \in \Omega^{0}$.

Since $\alpha$ could be positive or negative, for the optimal configuration we have

$v(\widehat{x})=0 \quad \forall \widehat{x} \in \Omega^{0}$.

However, since $v$ is harmonic, by the identity theorem of harmonic functions (Sarason 2007), we have that $v$ vanishes on the entire domain $\Omega$, and by ( 30 ) we have, for the optimal configuration:

$$
D_{T}^{1} \psi=0 \quad \text { in } \boldsymbol{\Omega} \text {. }
$$

Now we are ready to devise e topological derivativebased optimization algorithm to solve problem (37) based on the ideas in (Amstutz and Andrä 2006), that have been successfully applied to several problems. The procedure relies on a level-set domain representation of $\Theta^{+}$and $\Theta^{-}$ (Osher and Sethian 1988), and approximation of the topological optimality conditions through a fixed point iteration. The main difficulty of applying the ideas in (Amstutz and Andrä 2006) to the present case is that the first order topological derivative vanishes at the solution according to (40). The novelty of our algorithm is that it considers the expected variation of the objective functional given by the topological expansion (24) instead of the first order topological derivative to define the level sets.

With the adoption of a level-set domain representation, the region $\Theta^{+}$is characterized by a real valued function $\psi^{+}: \Theta \rightarrow \mathbb{R}$, belonging to $L^{2}(\Theta)$ :

$\boldsymbol{\Theta}^{+}=\left\{x \in \Theta, \psi^{+}(x)<0\right\}$.

Analogously, the region $\Theta^{-}$is characterized by the function $\psi^{-}$:

$\boldsymbol{\Theta}^{-}=\left\{x \in \Theta, \psi^{-}(x)<0\right\}$.

Let $E V(\widehat{x}, \varepsilon, \alpha)$ be the expected variation of the objective function of problem (37) for a perturbation of $j_{0}$ consisting in a circular region of current density $\alpha I$ of radius $\varepsilon$ and center $\widehat{x}$, namely,

$E V(\widehat{x}, \varepsilon, \alpha)=f_{1}(\varepsilon) D_{T}^{1} \psi(\widehat{x})+f_{2}(\varepsilon) D_{T}^{2} \psi(\widehat{x})$.

Take $\Theta^{0}=\Theta \backslash\left(\overline{\Theta^{+}} \cup \overline{\Theta^{-}}\right)$. A sufficient condition of local optimality for the class of perturbations considered is that the expected variation of the objective function be positive, i.e.,

$E V(\widehat{x}, \varepsilon,-1)>0, \quad \forall \widehat{x} \in \Theta^{+}$,

$E V(\widehat{x}, \varepsilon,+1)>0, \quad \forall \widehat{x} \in \Theta^{-}$,

$E V(\widehat{x}, \varepsilon, \pm 1)>0, \quad \forall \widehat{x} \in \Theta^{0}$.

Following Amstutz and Andrä (2006), to devise a levelset-based algorithm whose aim is to produce a topology that satisfies (44)-(46), we choose a value for $\varepsilon$ (in the numerical approach we define a mesh of cells in the domain $\Theta$ and take a value $\varepsilon$ related to the size of the cells) and define the functions

$g^{+}(x)=\left\{\begin{aligned}-E V(\widehat{x}, \varepsilon,-1) & \text { if } \widehat{x} \in \Theta^{+}, \\ E V(\widehat{x}, \varepsilon,+1) & \text { if } \widehat{x} \in \Theta^{0} \cup \Theta^{-},\end{aligned}\right.$

$g^{-}(x)=\left\{\begin{aligned}-E V(\widehat{x}, \varepsilon,+1) & \text { if } \widehat{x} \in \Theta^{-}, \\ E V(\widehat{x}, \varepsilon,-1) & \text { if } \widehat{x} \in \Theta^{0} \cup \Theta^{+} .\end{aligned}\right.$ 
With the above definitions and (41)-(42), it can be easily established that the sufficient conditions (44)-(46) are satisfied if the following equivalence relations between the functions $g^{+}$and $g^{-}$and the level-set functions $\psi^{+}$and $\psi^{-}$ hold

$\exists \tau^{+}>0 \quad$ s.t. $h\left(g^{+}\right)=\tau^{+} \psi^{+}$,

$\exists \tau^{-}>0$ s.t. $h\left(g^{-}\right)=\tau^{-} \psi^{-}$,

where $h: \mathbb{R} \rightarrow \mathbb{R}$ must be an odd and strictly increasing function, e.g.,

$h(x)=\operatorname{sign}(x)|x|^{\beta} \quad$ with $\beta>0$.

In fact, if $\widehat{x} \in \Theta^{+}$, then $\psi^{+}(\widehat{x})<0$. By (49) and since $h$ preserves the sign we have $g^{+}(\widehat{x})<0$, and by (47) we have $E V(\widehat{x}, \varepsilon,-1)>0$, proving (44). The proofs of (45) and (46) are analogous considering $\widehat{x}$ respectively in $\Theta^{-}$and $\Theta^{0}$.

Conditions (49)-(50) can be expressed equivalently as

$\theta^{+}:=\arccos \left[\frac{\left\langle h\left(g^{+}\right), \psi^{+}\right\rangle_{L^{2}(\Theta)}}{\left\|h\left(g^{+}\right)\right\|_{L^{2}(\Theta)}\left\|\psi^{+}\right\|_{L^{2}(\Theta)}}\right]=0$,

$\theta^{-}:=\arccos \left[\frac{\left\langle h\left(g^{-}\right), \psi^{-}\right\rangle_{L^{2}(\Theta)}}{\left\|h\left(g^{-}\right)\right\|_{L^{2}(\Theta)}\left\|\psi^{-}\right\|_{L^{2}(\Theta)}}\right]=0$,

where $\theta^{+}$is the angle between the vectors $h\left(g^{+}\right)$and $\psi^{+}$in $L^{2}(\Theta)$ and $\theta^{-}$is the counterpart between $h\left(g^{-}\right)$and $\psi^{-}$.

To find the optimal $\Theta^{+}$(the case of $\Theta^{-}$is completely analogous), we start from an initial level-set function $\psi_{0}^{+}$ which defines the initial guess. Then, the algorithm produces a sequence $\left(\psi_{i}^{+}\right)_{i \in \mathrm{N}}$ of level-set functions that provides successive approximations to the sufficient conditions of optimality. Starting from $\psi_{0}^{+}$, the sequence satisfies

$\psi_{n+1}^{+} \in \operatorname{co}\left(\psi_{n}^{+}, h\left(g_{n}^{+}\right)\right) \quad \forall n \in \mathbb{N}$,

where $\operatorname{co}\left(\psi_{n}^{+}, h\left(g_{n}^{+}\right)\right)$is the convex hull of $\left\{\psi_{n}^{+}, h\left(g_{n}^{+}\right)\right\}$ In the actual algorithm the initial guess $\psi_{0}^{+}$and subsequent iteration points can be normalized, see (Amstutz and Andrä 2006) for further details. The non-normalized sequences for both $\Theta^{+}$and $\Theta^{-}$are

$$
\left.\begin{array}{l}
\psi_{n+1}^{+}=\left(1-t_{n}\right) \psi_{n}^{+}+t_{n} h\left(g_{n}^{+}\right) \\
\psi_{n+1}^{-}=\left(1-t_{n}\right) \psi_{n}^{-}+t_{n} h\left(g_{n}^{-}\right)
\end{array}\right\} \quad \forall n \in \mathbb{N}
$$

where $t_{n} \in[0,1]$ is a step size determined by a line-search in order to decrease the value of the cost functional $J(\phi)$. The iterative process is stopped when certain criterion is satisfied, see Section 5. The angles $\theta^{+}$and $\theta^{-}$can be used as indicators of the accuracy of (49) and (50) at the final iteration and can be used to determine the necessity of a mesh refinement (Amstutz and Andrä 2006).

The next theorem shows that the algorithm given by the update rule (55) is well defined, i.e., for every $t_{n} \in[0,1]$ the functions $\psi_{n+1}^{+}$and $\psi_{n+1}^{-}$generate disjoint sets $\Theta^{+}$and $\Theta^{-}$. We prove by induction that $\psi_{n}^{+}+\psi_{n}^{-} \geq 0 \forall n \in \mathbb{N}$.
Table 1 Examples

\begin{tabular}{lllll}
\hline Example & NE & NC & SC & $I$ \\
\hline Ex1a & 120 & 4724 & 0.05 & 0.4 \\
Ex1b & 120 & 29520 & 0.02 & 0.4 \\
Ex2a & 169 & 4715 & 0.05 & 0.4 \\
Ex2b & 169 & 29463 & 0.02 & 0.4 \\
Ex3a & 152 & 10228 & 0.05 & 0.2 \\
Ex3b & 152 & 64120 & 0.02 & 0.2 \\
Ex4a & 172 & 11708 & 0.05 & 0.15 \\
Ex4b & 172 & 73420 & 0.02 & 0.15 \\
\hline
\end{tabular}

NE: number of boundary elements, NC: number of domain cells, SC: size of the cells

This latter inequality is sufficient to show that $\Theta^{+}$and $\Theta^{-}$ are disjoint, since a point $x$ in both sets would satisfy $\psi_{n}^{+}(x)<0$ and $\psi_{n}^{-}(x)<0$ and then would satisfy $\psi_{n}^{+}(x)+\psi_{n}^{-}(x)<0$.
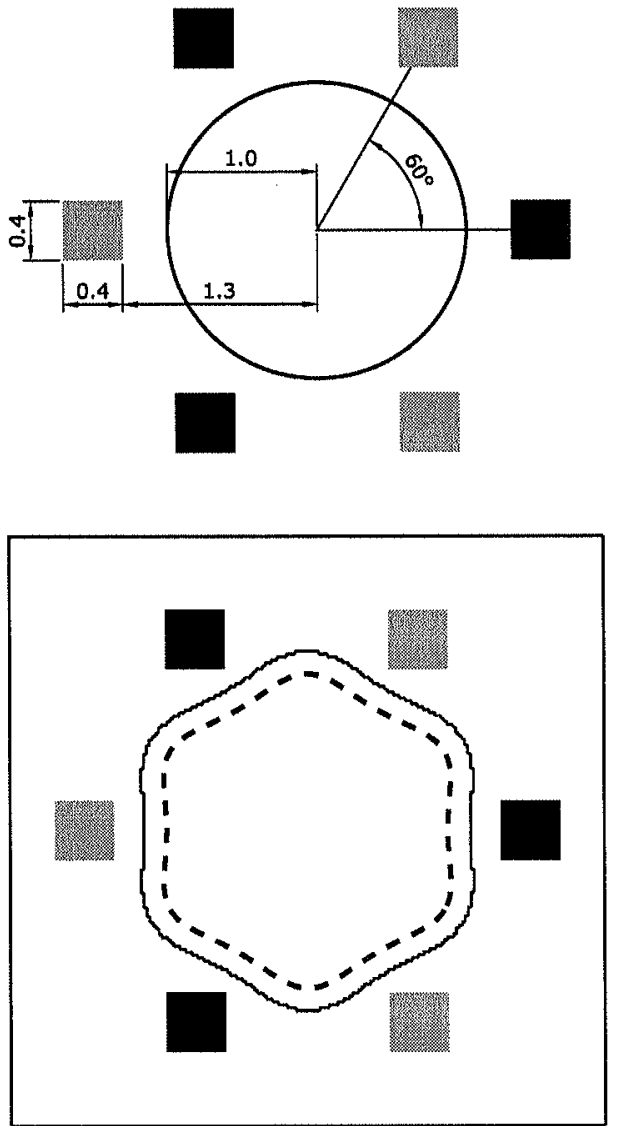

Fig. 1 Example 1. Top: initial configuration of the direct free-surface problem. Bottom target shape and exact solution. Black area: positive inductors, gray area: negative inductors, dashed line: target shape, thin solid line: boundary of the mesh of cells 


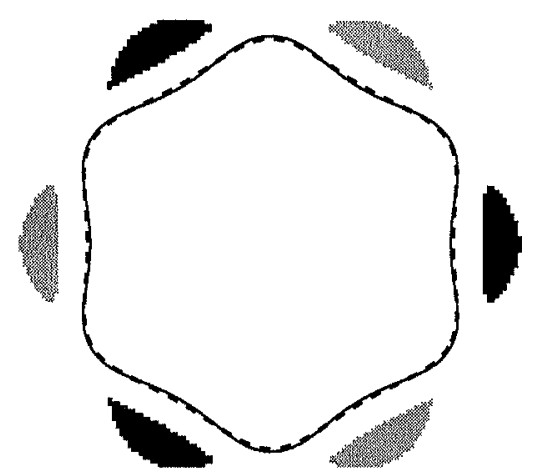

Fig. 2 Example 1. Solution for a mesh of cells of size 0.02 with $\beta=$ 3. Blackarea: positive inductors, gray area: negative inductors, dashed line: target shape, thin solid line: equilibrium shape

Theorem 3 Assume that $\psi_{0}^{+}+\psi_{0}^{-} \geq 0$. Then $\psi_{n}^{+}+\psi_{n}^{-} \geq$ $0 \forall n \in \mathbb{N}$.

Proof According to (47)-(48) and taking into account that $h$ is odd we have

$h\left(g_{n}^{+}(x)\right)+h\left(g_{n}^{-}(x)\right)=0 \quad \forall x \in \Theta^{+} \cup \Theta^{-}$.

In addition, for a point $x \in \Theta^{0}$ we have

$g_{n}^{+}(x)+g_{n}^{-}(x)=E V(x, \varepsilon,+1)+E V(x, \varepsilon,-1)$,

and, according to (43) and (30),

$g_{n}^{+}(x)+g_{n}^{-}(x)=2 f_{2}(\varepsilon) D_{T}^{2} \psi(x) \geq 0$,

hence $g_{n}^{+}(x) \geq-g_{n}^{-}(x)$ and, since $h$ is strictly increasing,

$h\left(g_{n}^{+}(x)\right) \geq h\left(-g_{n}^{-}(x)\right) \quad \forall x \in \Theta^{0}$.

Since $h$ is odd, the last inequality proves the desired result in $\Theta^{0}$ and thanks to (56) also in the whole domain $\Theta$ :

$h\left(g_{n}^{+}(x)\right)+h\left(g_{n}^{-}(x)\right) \geq 0 \quad \forall x \in \Theta$.

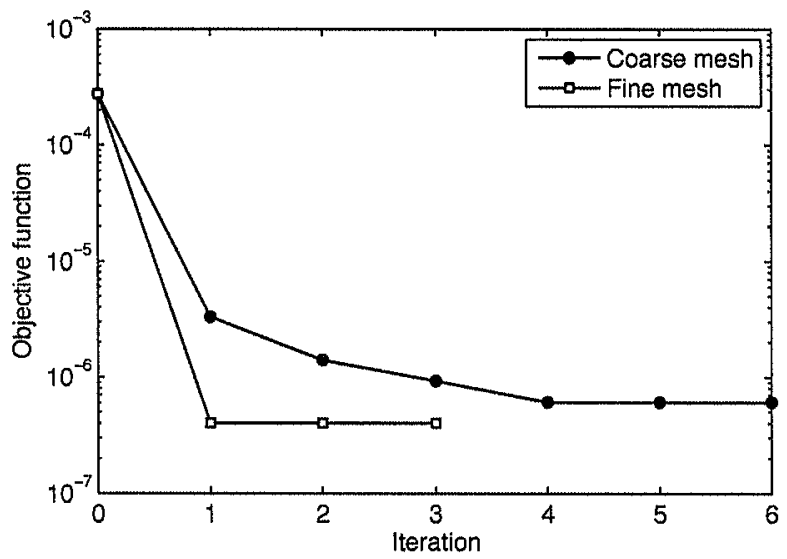

Fig. 3 Example 1. Evolution of the objective function for $\boldsymbol{\beta}=3$
The induction hypothesis is $\psi_{n}^{+}+\psi_{n}^{-} \geq 0$, and by (55) we have

$$
\psi_{n+1}^{+}+\psi_{n+1}^{-}=\left(1-t_{n}\right)\left[\psi_{n}^{+}+\psi_{n}^{-}\right]+t_{n}\left[h\left(g_{n}^{+}\right)+h\left(g_{n}^{-}\right)\right]
$$

Using the induction hypothesis and (60) we obtain $\psi_{n+1}^{+}+$ $\psi_{n+1}^{-} \geq 0$ for every $t_{n} \in[0,1]$.

Remark2 If there is no previous information about the optimum, a suitable initial guess that satisfies the inequality $\psi_{0}^{+}+\psi_{0}^{-} \geq 0$ is $\psi_{0}^{+}=\psi_{0}^{-}=1$, which corresponds to zero positive and negative currents.

\subsection{The asymmetric case}

In the asymmetric case, satisfaction of condition (2) cannot be ensured automatically by the algorithm described in the previous section. In fact, in Section 5 we present an example where the algorithm fails to find a solution satisfying (2).
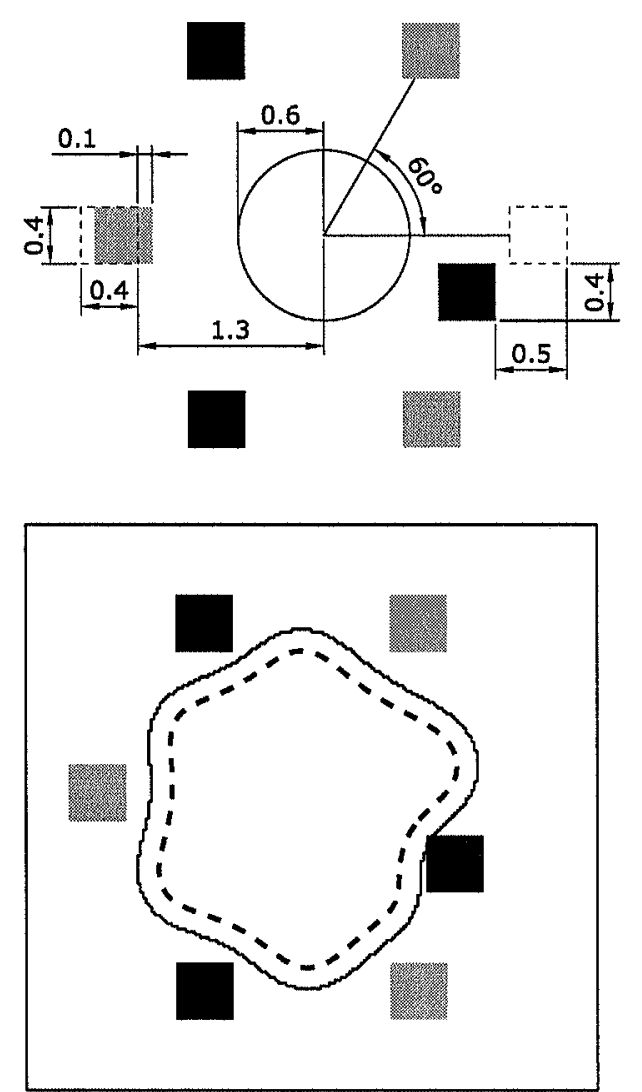

Fig. 4 Example 2. Top: initial configuration of the direct free-surface problem. Bottom target shape and exact solution. Blackarea: positive inductors, gray anea negative inductors, dashed line: target shape, thin solid line: boundary of the mesh of cells 


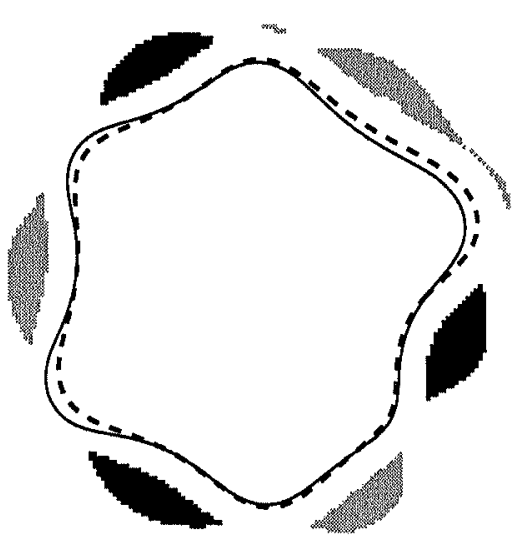

Fig. 5 Example 2. Solution for a mesh of cells of size 0.02 with $\beta=3$ and $\rho=1.0 \times 10^{-11}$. Black area: positive inductors, gray area: negative inductors, dashed line: target shape, thin solid line: equilibrium shape

In the asymmetric case we propose to relax this constraint and minimize the following penalty function:

$P(0)=J(\phi)+\frac{1}{2} \rho d\left(j_{0}\right)^{2}$,

where $\rho>0$ is a penalty parameter. Hence, in the penalty approach the current density $j_{0}$ is sought as the solution of the following optimization problem:

$\underset{j_{0} \in \mathrm{O}}{\operatorname{Minimize}} J(\phi)+\frac{1}{2} \rho d\left(j_{0}\right)^{2}$.

In this case, since $d\left(j_{\varepsilon}\right)=d\left(j_{0}\right)+|\Gamma|^{-1} \alpha \mu_{0} I \pi \varepsilon^{2}$, it is not difficult to see that $P$ admits the topological expansion (13), with the same functions $f_{1}$ and $f_{2}$ as $\psi$, and with the following topological derivatives:

$D_{T}^{1} P(\widehat{x})=D_{T}^{1} \psi(\widehat{x})+\rho \alpha \mu_{0} I|\Gamma|^{-1} d\left(j_{0}\right)$,

$D_{T}^{2} P(\widehat{x})=D_{T}^{2} \psi(\widehat{x})+\frac{1}{2} \rho \mu_{0}^{2} I^{2}|\Gamma|^{-2}$.

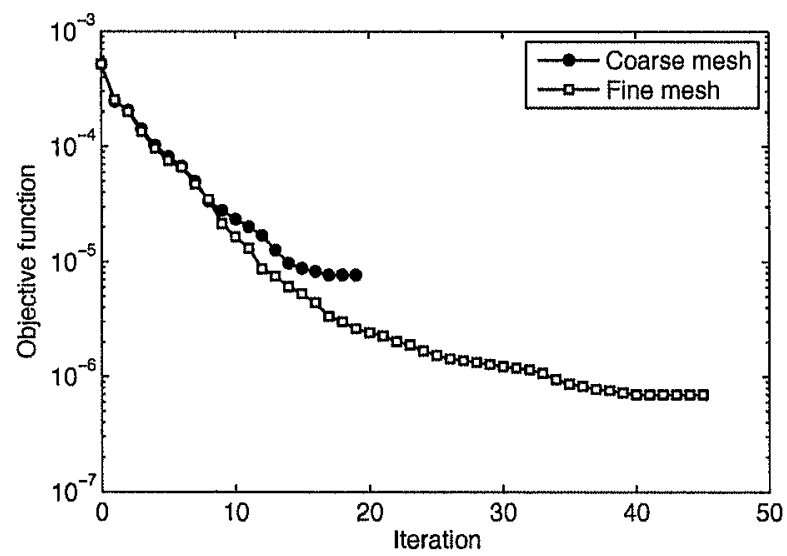

Fig. 6 Example 2. Evolution of the objective function for $\beta=3$ and $\rho=1 \times 10^{-11}$

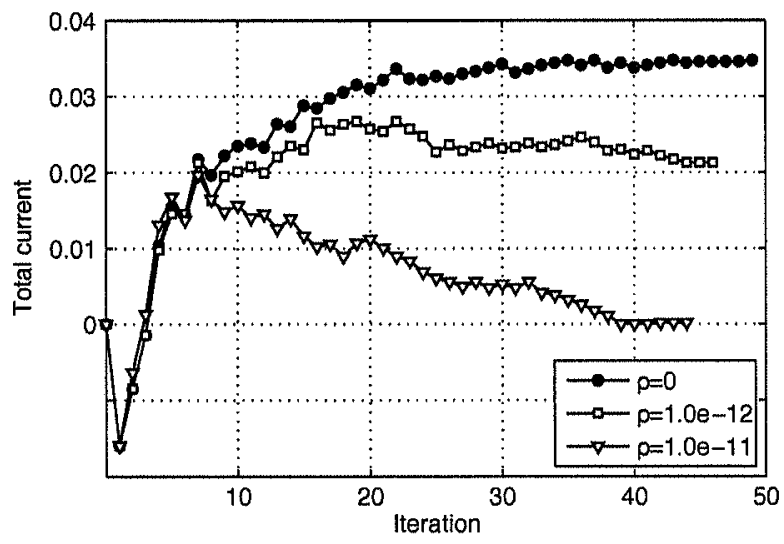

Fig. 7 Example 2. Evolution of the total current considering different values of $\rho$

Note that, as well as the symmetric case, the second order topological derivative $D_{T}^{2} P(\widehat{x})$ is strictly positive thanks to the strictly positive penalty term.
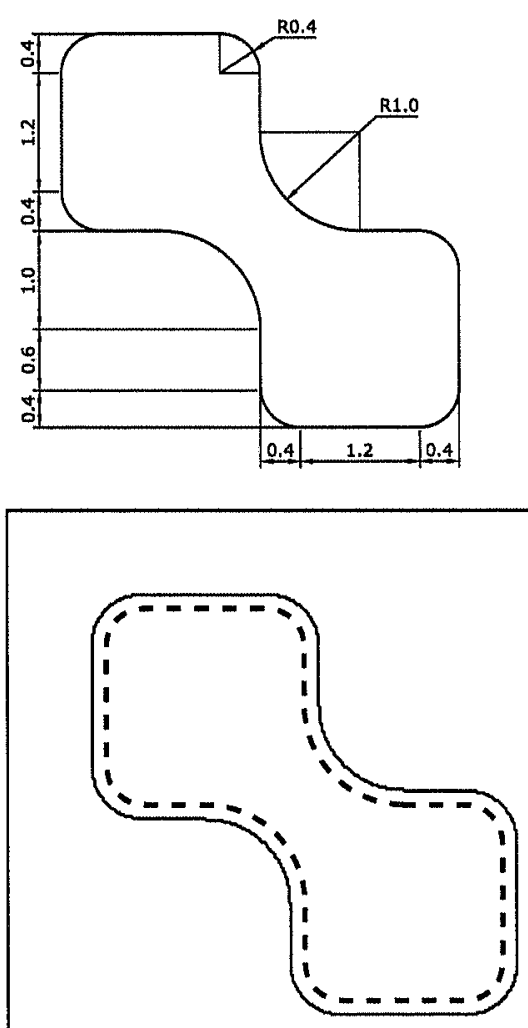

Fig. 8 Example 3. Top: description of the problem geometry. Bottom target shape. Dashed line: target shape, thin solid line: boundary of the mesh of cells. 


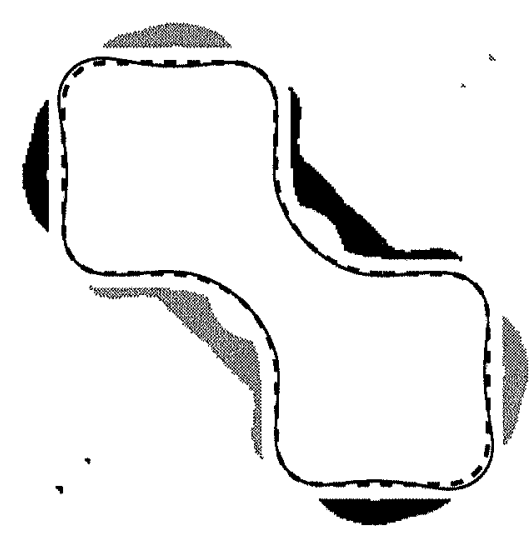

Fig. 9 Example 3. Solution for a mesh of cells of size 0.02 and $\beta=3$. Black area: positive inductors, gray area: negative inductors, dashed line: target shape, thin solid line: equilibrium shape.

The topology optimization procedure proposed to solve asymmetric problems is the same as in the previous section, using the penalty function (62) as objective and the expected variation $E V(x, \varepsilon, \alpha)$ computed according to the topological derivatives (64)-(65).

\section{Numerical examples}

Four examples are presented. The first two have known solutions, since the target shapes considered are equilibrium shapes for given current density distributions. The second example considers an asymmetric target shape, so that it serves to evaluate the effect of the penalty term proposed in Section 4.2. The last two examples consist of more realistic design problems. The boundary element method used in (Canelas et al. 2011) was applied here to solve the boundary value problems and to compute approximately the first and second order topological derivatives. In the examples

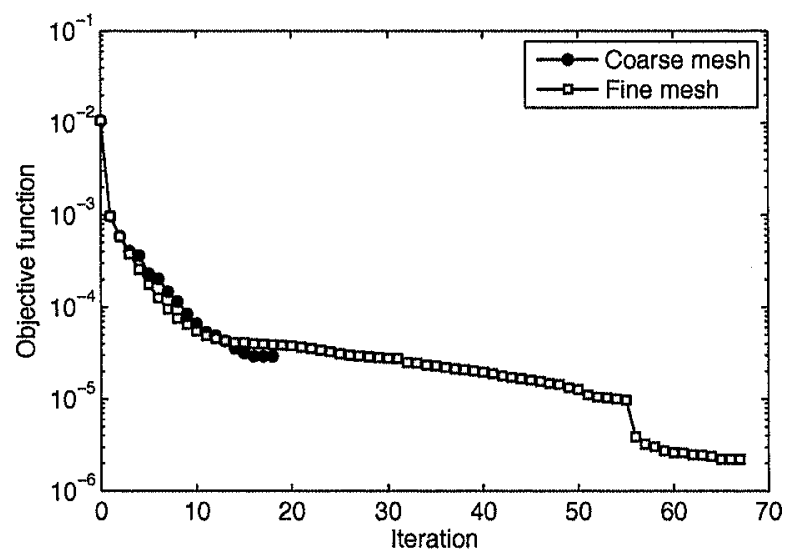

Fig. 10 Example 3. Evolution of the objective function for $\beta=3$. we express all physical magnitudes in the same consistent system of units. In this system we consider $\sigma=1.0 \times 10^{-4}$ and $\mu_{0}=1.0$. Two meshes are considered; the case (a) has cells of size 0.05 and the case (b) has cells of size 0.02 , see Table 1 . The optimization procedure stops when in subsequent iterations we have the same configuration of inductors, i.e., the level sets contains the same cells.

The target shape of the first example is the solution of a direct free-surface problem for a liquid metal column of cross-section area $S_{0}=\pi$, considering six distributed currents of density $I=0.4$ as shown in Fig. 1, see Fig. 1 . The result obtained for the finest mesh is shown in Fig. 2. The evolution of the objective function along the iterative process is shown in Fig. 3.

In the second example we move the inductors to generate an asymmetric objective shape as shown in Fig. 4. The
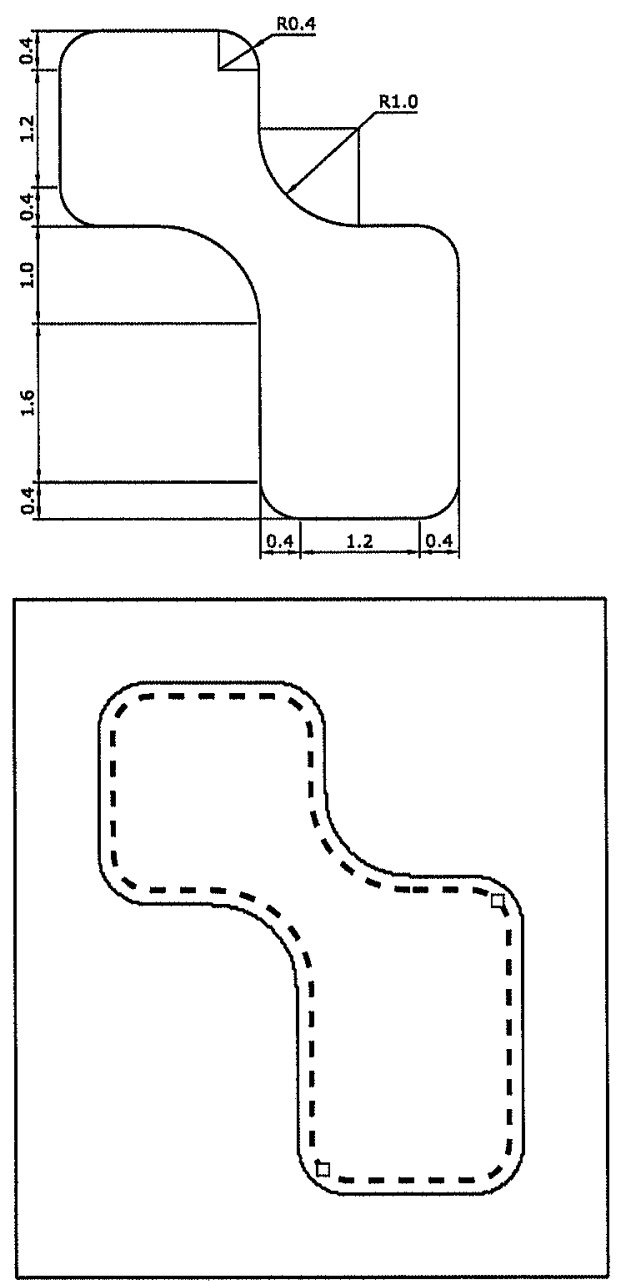

Fig. 11 Example 4. Top: description of the problem geometry. Bottom target shape. Dashed line: target shape, thin solid line: boundary of the mesh of cells, white squares points where the sign of $\varkappa$ remains unchanged 


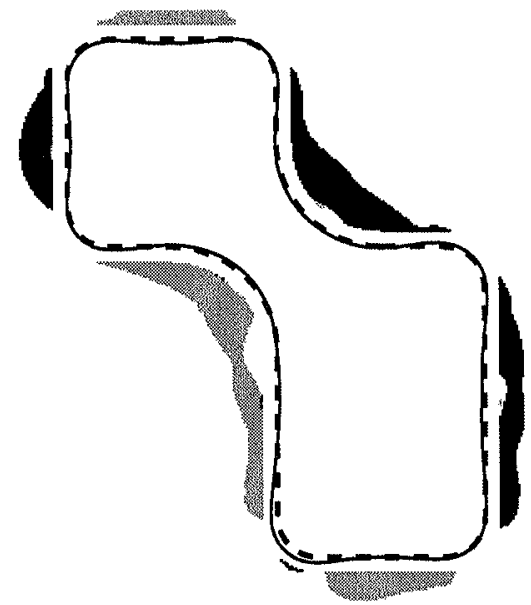

Fig. 12 Example 4. Solution for a mesh of cells of size 0.02 and $\beta=$ 3. Blackarea positive inductors, gray area negative inductors, dashed line: target shape, thin solid line: equilibrium shape

result obtained for the finest mesh is shown in Fig. 5. The evolution of the objective function along the iterative process is shown in Fig. 6 and the evolution of the total current considering three different values of the penalty parameter $\rho$ is shown in Fig. 7. Note that from the eighth iteration the effect of the penalty term is noticeable. For $\rho=0$ the total current never stop increasing, and for $\rho=1.0 \times 10^{-11}$ the total current converges to zero. The value $\rho=1.0 \times 10^{-12}$ is not large enough to produce a significant reduction of the total current.

The third example is depicted in Fig. 8. The result obtained for the finest mesh is shown in Fig. 9. The evolution of the objective function along the iterative process is shown in Fig. 10.

In the last example we consider the asymmetric target shape of Fig. 11. For this example, the compatibility Eq. (6)

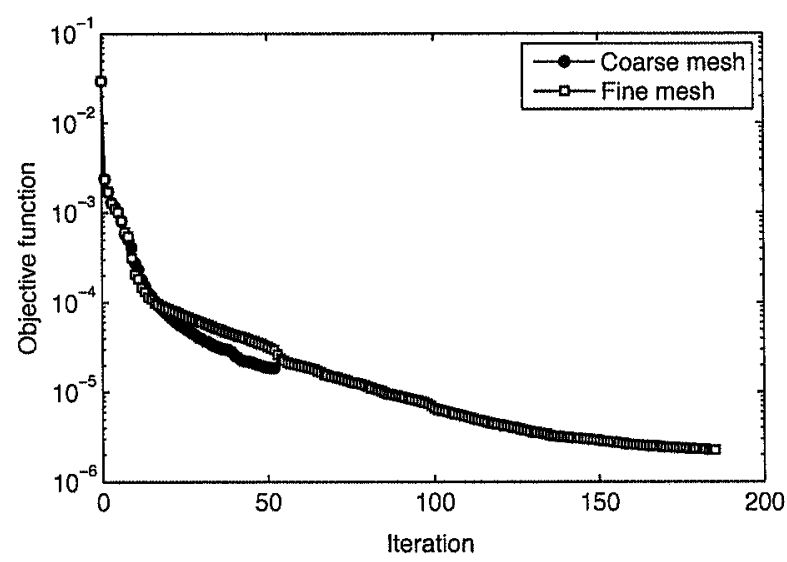

Fig. 13 Example 4. Evolution of the objective function for $\beta=3$

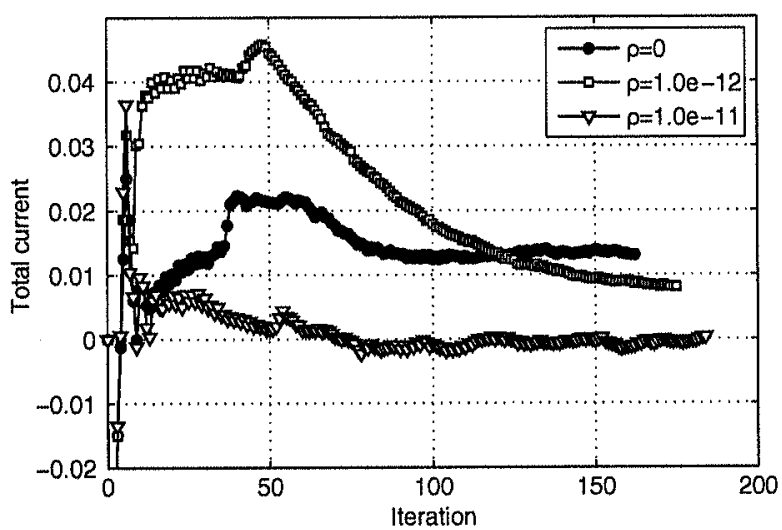

Fig. 14 Example 4. Evolution of the total current considering different values of $\rho$

is satisfied if the sign of $\varkappa$ does not change at the two vertices indicated in Fig. 11. The result obtained for the finest mesh is shown in Fig. 12. The evolution of the objective function along the iterative process is shown in Fig. 13 and the evolution of the total current considering three different values of the penalty parameter $\rho$ is shown in Fig. 14 . Note that the total current of the solution depends on $\rho$ as in Example 2. Solutions with almost zero total current are found for $\rho=1.0 \times 10^{-11}$.

\subsection{Results summary}

Table 2 resumes the information about the examples considered. For each example the number of iterations performed by the optimization algorithm proposed in (Canelas et al. 2011 ) is indicated, as well as those performed by the present algorithm for the values $\beta=1$ and $\beta=3$ in (51). In the case of the asymmetric examples, the results obtained for

Table 2 Results summary

\begin{tabular}{llllll}
\hline Example & IterP & Iter1 & Iter3 & IOF & FOF3 \\
\hline Ex1a & 24 & 4 & 6 & $2.747 \mathrm{e}-04$ & $6.088 \mathrm{e}-07$ \\
Ex1b & 47 & 4 & 3 & $2.747 \mathrm{e}-04$ & $4.009 \mathrm{e}-07$ \\
Ex2a & 21 & 17 & 19 & $5.187 \mathrm{e}-04$ & $7.698 \mathrm{e}-06$ \\
Ex2b & 63 & 61 & 45 & $5.187 \mathrm{e}-04$ & $6.977 \mathrm{e}-07$ \\
Ex3a & 29 & 18 & 18 & $1.066 \mathrm{e}-02$ & $2.936 \mathrm{e}-05$ \\
Ex3b & 108 & 134 & 67 & $1.066 \mathrm{e}-02$ & $2.219 \mathrm{e}-06$ \\
Ex4a & 53 & 56 & 52 & $2.976 \mathrm{e}-02$ & $4.248 \mathrm{e}-05$ \\
Ex4b & 145 & 190 & 184 & $2.976 \mathrm{e}-02$ & $2.257 \mathrm{e}-06$ \\
\hline
\end{tabular}

IterP: number of iterations performed by the algorithm proposed in (Canelas et al. 2011), Iter1: number of iterations performed by the level set algorithm for $\beta=1$, Iter3: number of iterations performed by the level set algorithm for $\beta=3$, IOF: initial value of the objective function, FOF3: final value of the objective function for $\beta=3$. 
$\rho=1.0 \times 10^{-11}$ are given. Table 2 shows that, for these examples, the present optimization algorithm with $\beta=3$ was generally the most efficient. Note that the solutions of Examples 2 and 4 required a relatively large number of iterations when compared to the similar symmetric Examples 1 and 3. However, for both asymmetric examples the penalty approach was effective to find suitable solutions.

\section{Conclusions}

We have proposed a new method for the topology design of inductors in electromagnetic casting. The method is based on a topology optimization formulation and uses level-sets together with first and second order topological derivatives to design suitable inductors.

The complete asymptotic expansion of the objective functional regarding the introduction of a small inductor was derived in this paper, generalizing the results of (Canelas et al. 2011). We have shown that the first order topological derivative vanishes at the solution, precluding the direct application of the topology optimization algorithm proposed by Amstutz and Andrä (2006) to the studied optimization problem. However, we have shown how to circumvent this difficulty using second order topological derivatives.

In the case of centrally symmetric geometries, the method proposed generates a sequence of solutions satisfying all the equality constraints. A penalty-based approach was proposed for problems with asymmetric geometries.

The set of examples considered show that the method proposed is effective and efficient, and therefore can be successfully used in the design of inductors in electromagnetic casting.

Acknowledgments The authors thank the Brazilian Research Councils CAPES, CNPq and FAPERJ, the Uruguayan Councils ANII and CSIC and the French Research Councils COFECUB, INRIA and CNRS for the financial support.

\section{References}

Ammari H, Kang H (2004) Reconstruction of small inhomogeneities from boundary measurements. Lectures Notes in Mathematics, vol 1846. Springer-Verlag, Berlin

Ammari H, Kang $\mathrm{H}$ (2007) Polarization and moment tensors with applications to inverse problems and effective medium theory. Applied Mathematical Sciences, vol 162. Springer-Verlag, New York

Amstutz S ((2006)) Sensitivity analysis with respect to a local perturbation of the material property. Asymptot Anal 49(1-2):87-108

Amstutz S, Andrä H (2006) A new algorithm for topology optimization using a level-set method. J Comput Phys 216(2):573-588. doi:10.1016/j.jcp.2005.12.015
Amstutz S, Novotny AA (2010) Topological optimization of structures subject to von Mises stress constraints. Struc Multidiscip Optim 41(3):407-420. doi:10.1007/s00158-009-0425-x

Amstutz S, Horchani I, Masmoudi M (2005) Crack detection by the topological gradient method. Control Cybern 34(1):81-101

Amstutz S, Giusti SM, Novotny AA (2010) Topological derivative in multi-scale linear elasticity models applied to the synthesis of microstructures. Int J Numer Methods Eng 84(6):733-756. doi: $10.1002 /$ nme. 2922

Atkinson KE (1997) The numerical solution of integral equation of the second kind, Cambridge monographs on applied and computational mathematics, vol 4. Cambridge University Press, Cambridge doi:10.1017/CBO9780511626340

Belaid LJ, Jaoua M, Masmoudi M, Siala L (2008) Application of the topological gradient to image restoration and edge detection. Eng Anal Bound Elem 32(11):891-899. doi:10.1016/j.enganabound, 2008.01 .004

Brancher JP, Séro-Guillaume OE (1985) Étude de la déformation d'un liquide magnétique. Arch Ratio Mechan Anal 90(1):57-85. doi: $10.1007 / \mathrm{BF} 00281587$

Brühl M, Hanke M, Vogelius MS (2003) A direct impedance tomography algorithm for locating small inhomogeneities. Numer Math 93(4):635-654. doi: $10.1007 / \mathrm{s} 002110200409$

Canelas A, Roche JR, Herskovits J (2009a) Inductor shape optimization for electromagnetic casting. Struct Multidiscip Optim 39(6):589-606. doi: 10.1007/s00158-009-0386-0

Canelas A, Roche JR, Herskovits J (2009b) The inverse electromagnetic shaping problem. Struct Multidiscip Optim 38(4):389-403. doi: $10.1007 / \mathrm{s} 00158-008-0285-9$

Canelas A, Novotny AA, Roche JR (2011) A new method for inverse electromagnetic casting problems based on the topological derivative. J Comput Phys 230(9):3570-3588, doi:10.1016/ j.jcp.2011.01.049

Canelas A, Novotny A, Roche JR (2012) Topological derivatives and a level set approach for an inverse electromagnetic casting problem. In: World congress of computational mechanics. São Paulo, Brazil

Céa J, Garreau S, Guillaume P, Masmoudi M (2000) The shape and topological optimizations connection. Comput Methods Appl Mech Eng 188(4):713-726

Eppler K, Harbrecht H (2010) On a kohn-vogelius like formulation of free boundary problems. Comput Optim Appl Online. doi: $10.1007 / \mathrm{s} 10589-010-9345-3$

Eschenauer H, Kobelev V, Schumacher A (1994) Bubble method for topology and shape optmization of structures. Struct Optimi $8(1): 42-51$

de Faria JR, Novotny AA (2009) On the second order topologial asymptotic expansion. Struct Multidis Optim 39(6):547-555. doi: $10.1007 / \mathrm{s} 00158-009-0436-7$

Feijó GR (2004) A new method in inverse scattering based on the topological derivative. Inverse Prob 20(6):1819-1840. doi: 10.1088/0266-5611/20/6/008

Felici TP, Brancher JP (1991) The inverse shaping problem. Eur J Mech B Fluids 10(5):501-512

Friedman A, Vogelius M (1989) Identification of small inhomogeneities of extreme conductivity by boundary measurements: a theorem on continuous dependence. Arch Ration Mech Anal 105(4):299-326. doi:10.1007/BF00281494

Fu HZ, Shen J, Liu L, Hao QT, Li SM, Li JS (2004) Electromagnetic shaping and solidification control of Ni-base superalloys under vacuum. J Mater Process Tech 148(1):25-29. doi:10.1016/ j.jmatprotec. 2003.11.039

Gagnoud A, Etay J, Garnier M (1986) Le problème de frontière libre en lévitation électromagnétique. J de Mécan Théor et Appl 5(6):911934 
Garreau S, Guillaume P, Masmoudi M (2001) The topological asymptotic for PDE systems: the elasticity case. SIAM J Control Optim 39(6):1756-1778. doi:10.1137/\$0363012900369538

Giroire J (1976) Formulation variationnelle par équations intégrales de problèmes aux limites extérieurs. Tech. Rep. 6, Centre de Mathématiques Appliquées de l'Ecole Polytechnique

Guzina BB, Bonnet M (2006) Small-inclusion asymptotic of misfit functionals for inverse problems in acoustics. Inverse Prob 22(5):1761-1785. doi:10.1088/0266-5611/22/5/014

Henrot $A$, Pierre $M$ (1989) Un problème inverse en formage des métaux liquides. RAIRO Modél Math Anal Num 23(1):155177

Hintermüller M, Laurain A (2008) Electrical impedance tomography: from topology to shape. Control Cybern 37(4):913933

Hintermüller M, Laurain A (2009) Multiphase image segmentation and modulation recovery based on shape and topological sensitivity. J Math Imaging Vis 35:1-22. doi:10,1007/s10851-0090150-5

Hintermüller M, Laurain A, Novotny AA (2012) Second-order topological expansion for electrical impedance tomography. Adv Comput Math 36(2):235-265. doi: $10.1007 / \mathrm{s} 10444-011-9205.4$

Hsiao G, Wendland WL (2008) Boundary integral equations, applied mathematical sciences, vol 164. Springer-Verlag, Berlin

Jackson JD (1998) Classical electrodynamics, 3rd edn. Wiley

Kohn R, Vogelius M (1984) Determining conductivity by boundary measurements. Commun Pure Appl Math 37(3):289-298. doi: $10.1002 / \mathrm{cpa} .3160370302$

Larrabide I, Feijóo RA, Novotny AA, Taroco E (2008) Topological derivative: a tool for image processing. Comput Struct 86 (13-14):1386-1403. doi: 10.1016/j.compstruc.2007.05.004

Moffatt HK (1985) Magnetostatic equilibria and analogous Euler flows of arbitrarily complex topology. Part 1. Fundam J Fluid Mech 159:359-378. doi: $10.1017 /$ S0022112085003251

Nazarov SA (2003) Asymptotic analysis of shape functionals. J Math Pures Appl 82(2):125-196. doi: 10.1016/S0021-7824(03)00004-7

Nédélec JC (2001) Acoustic and Electromagnetic Equations. Integral representations for harmonic problems, Applied Mathematical Sciences, vol 144. Springer-Verlag, New York

Novotny AA, Sokołowski J (2013) Topological derivatives in shape optimization. Interaction of Mechanics and Mathematics, Springer

Novotny AA, Feijóo RA, Taroco E, Padra C (2007) Topological sensitivity analysis for three-dimensional linear elasticity problem. Comput Methods Appl Mech Eng 196(41-44):4354 4364. doi: 10.1016 /j.cma.2007.05.006

Novruzi A, Roche JR (1995) Second order derivatives, Newton method, application to shape optimization. Tech. Rep. RR-2555, INRIA

Novruzi A, Roche JR (2000) Newton's method in shape optimisation: a three-dimensional case. BIT Numer Math 40(1):102-120. doi:10.1023/A:1022370419231

Osher S, Sethian J (1988) Front propagating with curvature dependent speed: algorithms based on Hamilton-Jacobi formulations. J Comput Phys 78:12-49. doi:10.1016/0021-9991(88)90002-2

Pierre M, Roche JR (1991) Computation of free surfaces in the electromagnetic shaping of liquid metals by optimization algorithms. Eur J Mech B Fluids 10(5):489-500

Pierre M, Roche JR (1993) Numerical simulation of tridimensional electromagnetic shaping of liquid metals. Numer Math 65(1):203217. doi:10.1007/BF01385748

Roche JR (1997) Gradient of the discretized energy method and discretized continuous gradient in electromagnetic shaping simulation. Appl Math Comput Sci 7(3):545-565

Roche JR (2005) Adaptive Newton-like method for shape optimization. Control Cybern 34(1):363-377

Roche JR (1996) Numerical methods for shape identification problems. Control Cybern 25(5):867-894. special issue: Shape Optimization and Scientific Computations

Sarason D (2007) Complex function theory, 2nd edn. American Mathematical Society, Providence, RI

Shercliff JA (1981) Magnetic shaping of molten metal columns. Proceedings of the Royal Society of London Series A. Math Phys Sci 375(1763):455-473. doi:10.1098/rspa.1981.0063

Shin J, Spicer JP, Abell JA (2012) Inverse and direct magnetic shaping problems. Struct Multidiscip Optim 46(2):285-301. doi: $10.1007 / \mathrm{s} 00158-011-0756-2$

Sokołowski J, Żochowski A (1999) On the topological derivative in shape optimization. SIAM J Control Optim 37(4):1251-1272. doi: $10.1137 /$ S0363012997323230

Sokołowski J, Żochowski A (2003) Optimality conditions for simultaneous topology and shape optimization. SIAM J Control Optim 42(4):1198-1221. doi:10.1137/S0363012901384430

Zhiqiang C, Fei J, Xingguo Z, Hai H, Junze J (2002) Microstructures and mechanical characteristics of electromagnetic casting and direct-chill casting 2024 aluminum alloys. Mater Sci Eng A 327(2):133-137. doi:10.1016/\$0921-5093(01)01673-2 